\title{
A note on double Laplace decomposition method and nonlinear partial differential equations
}

\author{
Hassan Eltayeb
}

Mathematics Department, College of Science, King Saud University, Riyadh, Saudi Arabia

Received: 2 August 2017, Accepted: 21 September 2017

Published online: 8 November 2017.

\begin{abstract}
In this article we propose a new technique, namely double Laplace decomposition method for solving nonlinear partial differential equation. The technique is described and illustrated with some examples.
\end{abstract}

Keywords: Double Laplace transform, inverse laplace transform, nonlinear partial differential equation.

\section{Introduction}

The linear and nonlinear problems, that are appear in many areas of scientific research such as solid state physics, wave equation, telegraph equation, plasma physics, fluid mechanics, which are modeled by linear and nonlinear partial differential equations. Also the double Laplace transform and some of its application are used to solve general linear telegraph equation and wave equation with initial and boundary conditions see [3]. Also double Laplace transform applied by Eltayeb and Kilicman [4] and [5] to solved non-homogeneous wave equation with variable coefficients. In this work we use the double Laplace decomposition methods to solve nonlinear partial differential equation. In special cases four examples are given. We are recalling the following definitions which are given by [2]. the double Laplace transform defined as

$$
L_{x} L_{t}[f(x, s)]=\quad F(p, s)=\int_{0}^{\infty} e^{-p x} \int_{0}^{\infty} e^{-s t} f(x, t) d t d x
$$

where $x, t>0$ and $p, s$ complex value and further double Laplace transform of the first order partial derivatives are given by

$$
L_{x} L_{t}\left[\frac{\partial f(x, t)}{\partial x}\right]=p F(p, s)-F(0, s) .
$$

Similarly the double Laplace transform for second partial derivative with respect to $x$ and $t$ are defined as follows

$$
\begin{aligned}
& L_{x x}\left[\frac{\partial^{2} f(x, t)}{\partial^{2} x}\right]=p^{2} F(p, s)-p F(0, s)-\frac{\partial F(0, s)}{\partial x} \\
& L_{t t}\left[\frac{\partial^{2} f(x, t)}{\partial^{2} t}\right]=s^{2} F(p, s)-s F(p, 0)-\frac{\partial F(p, 0)}{\partial t}
\end{aligned}
$$


Theorem 1. Let $f(x, t)$ and $g(x, t)$ be having double Laplace transform. Then double Laplace transform of the double convolution of the $f(x, t)$ and $g(x, t)$,

$$
f(x, t) * * g(x, t)=\int_{0}^{t} \int_{0}^{x} f(x-\eta, t-\zeta) g(\eta, \zeta) d \eta d \zeta
$$

is given by

$$
L_{x} L_{t}[f(x, s) * * g(x, t) ; p, s]=F(p, s) G(p, s)
$$

Proof. By applying the definition of double Laplace transform and double convolutions, we get

$$
\begin{aligned}
L_{x} L_{t}[f(x, s) * * g(x, t) ; p, s] & =\int_{0}^{\infty} \int_{0}^{\infty} e^{-p x-s t}(f(x, t) * * g(x, t)) d t d x \\
& =\int_{0}^{\infty} \int_{0}^{\infty} e^{-p x-s t}\left(\int_{0}^{t} \int_{0}^{x} f(x-\eta, t-\zeta) g(\eta, \zeta) d \eta d \zeta\right) d x d t
\end{aligned}
$$

let $\alpha=x-\eta$ and $\beta=t-\zeta$, and the region of integration becomes as $\eta \geq 0, \zeta \geq 0$ and $\alpha \geq 0, \beta \geq 0$, we obtain

$$
L_{x} L_{t}[f(x, s) * * g(x, t) ; p, s]=\left(\int_{0}^{\infty} \int_{0}^{\infty} e^{-p \eta-s \zeta} f(\eta, \zeta) d \eta d \zeta\right)\left(\int_{0}^{\infty} \int_{0}^{\infty} e^{-p \alpha-s \beta} g(\alpha, \beta) d \alpha d \beta\right) .
$$

Then, one can see that

$$
L_{x} L_{t}[f(x, s) * * g(x, t) ; p, s]=F(p, s) G(p, s)
$$

In the next theorem we study the nonlinear partial differential equation with convolution operator by using double Laplace decomposition methods

Theorem 2. Consider the nonlinear partial differential equation with convolution term as follows

$$
\begin{aligned}
\frac{\partial^{2} u(x, t)}{\partial^{2} x}+R u(x, t)+K u(x, t) & =g(x, t) * * h(x, t) \\
u(0, t) & =f_{1}(t), \frac{\partial u(0, t)}{\partial x}=f_{2}(t)
\end{aligned}
$$

$R$ represents a linear operator and $K$ denoted by nonlinear differential operator with convolution as follows

$$
K u(x, t)=(u(x, t))^{n} * *\left(\frac{\partial^{2} u(x, t)}{\partial^{2} t}\right)^{m}
$$

then the solution of $E q(2)$ given by

$$
\left(\sum_{n=0}^{\infty} u_{n}(x, t)\right)=f_{1}(t)+x f_{2}(t)-L_{p}^{-1} L_{s}^{-1}\left[\frac{1}{p^{2}} L_{x} L_{t}\left[R \sum_{n=0}^{\infty} u_{n}(x, t)+\sum_{n=0}^{\infty} A_{n}\right]\right]+L_{p}^{-1} L_{s}^{-1}\left[\frac{1}{p^{2}} L_{x} L_{t}[g(x, t) * * h(x, t)]\right]
$$

where $\sum_{n=0}^{\infty} A_{n}=K u(x, t)$.

Proof. By taking double Laplace transform for both sides of $\mathrm{Eq}(2)$ and single Laplace transform for $\mathrm{Eq}(3)$ we obtain

$$
U(p, s)=\frac{F_{1}(s)}{p}+\frac{F_{2}(s)}{p^{2}}-\frac{1}{p^{2}} L_{x} L_{t}[R u(x, t)+K u(x, t)]+\frac{1}{p^{2}} L_{x} L_{t}[[g(x, t) * * h(x, t)]] .
$$


On using double inverse Laplace transform for $\mathrm{Eq}(5)$ we have

$$
u(x, t)=f_{1}(t)+x f_{2}(t)-L_{p}^{-1} L_{s}^{-1}\left[\frac{1}{p^{2}} L_{x} L_{t}[R u(x, t)+K u(x, t)]\right]+L_{p}^{-1} L_{s}^{-1}\left[\frac{1}{p^{2}} L_{x} L_{t}[g(x, t) * * h(x, t)]\right],
$$

applying the decomposition method, then we consider the solution as an infinite series given as follows

$$
u(x, t)=\sum_{n=0}^{\infty} u_{n}(x, t)
$$

The nonlinear operator is decompose as follows

$$
K u(x, t)=\sum_{n=0}^{\infty} A_{n}
$$

Where $A_{n}$ are Adomian polynomials given by

$$
A_{n}=\frac{1}{n !} \frac{d^{n}}{d \lambda^{n}}\left[N\left(\sum_{i=0}^{\infty} \lambda^{i} u_{i}\right)\right]_{\lambda=0}, \quad n=0,1,2, \ldots
$$

By subsitituting $\mathrm{Eq}(7)$ and $\mathrm{Eq}(8)$ into $\mathrm{Eq}(6)$ we complete the proof.

The purpose of this parts is to study the use of modified double Laplace transform algorithm for the nonlinear partial differential equations. We consider the general form of second order nonhomogeneous nonlinear partial differential equations with initial conditions is given below

$$
L u(x, t)+R u(x, t)+N u(x, t)=h(x, t)
$$

with initial condition

$$
u(0, t)=f(t), \quad u_{x}(0, t)=g(t),
$$

where $L$ denoted by differential operator $L=\frac{\partial^{2}}{\partial x^{2}}, R$ is called linear operator, $N u$ represents a general non-linear differential operator and $h(x, t)$ is source term. The methodology consists of applying double Laplace transform on both sides of $\mathrm{Eq}(10)$

$$
L_{x} L_{t}[L u(x, t)+R u(x, t)+N u(x, t)=h(x, t)]
$$

The frist step we applying the differentiation property of double and single Laplace transform we get

$$
U(p, s)=\frac{F(s)}{p}+\frac{G(s)}{p^{2}}-\frac{1}{p^{2}} L_{x} L_{t}[R u(x, t)+N u(x, t)]+\frac{1}{p^{2}} L_{x} L_{t}[h(x, t)]
$$

The second step in Laplace decomposition method is that we represent solution as an infinite series given below

$$
u(x, t)=\sum_{n=0}^{\infty} u_{n}(x, t),
$$

The nonlinear operator is decompose as follows

$$
N u=\sum_{n=0}^{\infty} A_{n}
$$


where $A_{n}$ are Adomian polynomials given by $\mathrm{Eq}(9)$. By subsitituting $\mathrm{Eq}(14)$ and $\mathrm{Eq}(15)$ into $\mathrm{Eq}(13)$ we obtain

$$
L_{x} L_{t}\left(\sum_{n=0}^{\infty} u_{n}(x, t)\right)=\frac{F(s)}{p}+\frac{G(s)}{p^{2}}-\frac{1}{p^{2}} L_{x} L_{t}\left[R \sum_{n=0}^{\infty} u_{n}(x, t)+\sum_{n=0}^{\infty} A_{n}\right]+\frac{1}{p^{2}} L_{x} L_{t}[h(x, t)]
$$

Now, applying the inverse double Laplace transform on both sides of Eq(16), we get

$$
\left(\sum_{n=0}^{\infty} u_{n}(x, t)\right)=f(t)+x g(t)-L_{p}^{-1} L_{s}^{-1}\left[\frac{1}{p^{2}} L_{x} L_{t}\left[R \sum_{n=0}^{\infty} u_{n}(x, t)+\sum_{n=0}^{\infty} A_{n}\right]\right]+L_{p}^{-1} L_{s}^{-1}\left[\frac{1}{p^{2}} L_{x} L_{t}[h(x, t)]\right] .
$$

On comparing both sides of the $\mathrm{Eq}(17)$ we have

$$
\begin{aligned}
& u_{0}(x, t)=f(t)+x g(t)+L_{p}^{-1} L_{s}^{-1}\left[\frac{1}{p^{2}} L_{x} L_{t}[h(x, t)]\right] \\
& u_{1}(x, t)=L_{p}^{-1} L_{s}^{-1}\left[\frac{1}{p^{2}} L_{x} L_{t}\left[R u_{0}(x, t)+A_{0}\right]\right] \\
& u_{2}(x, t)=L_{p}^{-1} L_{s}^{-1}\left[\frac{1}{p^{2}} L_{x} L_{t}\left[R u_{1}(x, t)+A_{1}\right]\right]
\end{aligned}
$$

In general the recursive relation is given by

$$
u_{n+1}(x, t)=-L_{p}^{-1} L_{s}^{-1}\left[\frac{1}{p^{2}} L_{x} L_{t}\left[R u_{n}(x, t)+A_{n}\right]\right], n \geq 0 .
$$

Now first of all we applying double Laplace transform of the terms on the right hand side of Eq(21) then applying inverse double Laplace transform we get the values of $u_{1}, u_{2}, \ldots, u_{n}$ respectively

\section{Applications}

To demonstrate the applicability of the above-presented method, for nonlinear partial differential equations, we now consider some examples.

Example 1.We Consider the nonlinear partial differential equation

$$
u_{x x}+\left(u_{t}\right)^{2}+u-u^{2}=-x e^{-t}
$$

with initial conditions

$$
u(0, t)=0, \quad u_{x}(0, t)=e^{-t}
$$

On using double and single laplace transform method we obtain

$$
U(p, s)=\frac{1}{p^{2}(s+1)}+\frac{1}{p^{4}(s+1)}-\frac{1}{p^{2}} L_{x} L_{t}\left[\left(u_{t}\right)^{2}\right]+\frac{1}{p^{2}} L_{x} L_{t}\left[u^{2}\right]-\frac{1}{p^{2}} L_{x} L_{t}[u]
$$

By taking double inverse Laplace transform, we have

$$
u(x, t)=x e^{-t}+\frac{x^{3} e^{-t}}{6}-L_{p}^{-1} L_{s}^{-1}\left(\frac{1}{p^{2}} L_{x} L_{t}\left[\left(u_{t}\right)^{2}\right]\right)+L_{p}^{-1} L_{s}^{-1}\left(\frac{1}{p^{2}} L_{x} L_{t}\left[u^{2}\right]\right)-L_{p}^{-1} L_{s}^{-1}\left(\frac{1}{p^{2}} L_{x} L_{t}[u]\right)
$$


Applying Eq(17) we get

$$
\sum_{n=0}^{\infty} u_{n}(x, t)=x e^{-t}-\frac{x^{3} e^{-t}}{6}-L_{p}^{-1} L_{s}^{-1}\left[\frac{1}{p^{2}} L_{x} L_{t}\left[\sum_{n=0}^{\infty} A_{n}\right]\right]+L_{p}^{-1} L_{s}^{-1}\left[\frac{1}{p^{2}} L_{x} L_{t}\left[\sum_{n=0}^{\infty} B_{n}\right]\right]-L_{p}^{-1} L_{s}^{-1}\left[\frac{1}{p^{2}} L_{x} L_{t}\left[\sum_{n=0}^{\infty} u_{n}\right]\right]
$$

where $A_{n}$ and $B_{n}$ are Adomian polynomials that represent by

$$
\left(u_{t}\right)^{2}=\sum_{n=0}^{\infty} A_{n}, u^{2}=\sum_{n=0}^{\infty} B_{n}
$$

The beginning terms of Adomian polynomials, which is given by

$$
\begin{aligned}
& A_{0}=\left(u_{0}\right)_{t}^{2}, A_{1}=2\left(u_{0}\right)_{t}\left(u_{1}\right)_{t}, A_{2}=\left(u_{1}\right)_{t}^{2}+2\left(u_{0}\right)_{t}\left(u_{2}\right)_{t} \\
& B_{0}=u_{0}^{2}, B_{1}=2 u_{0} u_{1} B_{2}=u_{1}^{2}+2 u_{0} u_{2} .
\end{aligned}
$$

By using $\mathrm{Eq}(18), \mathrm{Eq}(21)$ and $\mathrm{Eq}(26)$ we get

$$
\begin{aligned}
u_{0}(x, t) & =x e^{-t}+\frac{x^{3} e^{-t}}{6} \\
u_{n+1}(x, t) & =-L_{p}^{-1} L_{s}^{-1}\left[\frac{1}{p^{2}} L_{x} L_{t}\left[\sum_{n=0}^{\infty} A_{n}\right]\right]+L_{p}^{-1} L_{s}^{-1}\left[\frac{1}{p^{2}} L_{x} L_{t}\left[\sum_{n=0}^{\infty} B_{n}\right]\right]-L_{p}^{-1} L_{s}^{-1}\left[\frac{1}{p^{2}} L_{x} L_{t}\left[\sum_{n=0}^{\infty} u_{n}\right]\right]
\end{aligned}
$$

Applying above recursive relation, we obtain

$$
\begin{aligned}
u_{1}(x, t) & =-L_{p}^{-1} L_{s}^{-1}\left[\frac{1}{p^{2}} L_{x} L_{t}\left[x e^{-t}+\frac{x^{3} e^{-t}}{6}\right]\right] \\
& =-L_{p}^{-1} L_{s}^{-1}\left[\frac{1}{p^{4}(s+1)}+\frac{1}{p^{6}(s+1)}\right] \\
& =-\frac{x^{3} e^{-t}}{6}-\frac{1}{5 !} x^{5} e^{-t}
\end{aligned}
$$

and

$$
\begin{aligned}
u_{2}(x, t) & =-L_{p}^{-1} L_{s}^{-1}\left[\frac{1}{p^{2}} L_{x} L_{t}\left[-\frac{x^{3} e^{-t}}{6}-\frac{1}{5 !} x^{5} e^{-t}\right]\right] \\
& =-L_{p}^{-1} L_{s}^{-1}\left[-\frac{1}{p^{6}(s+1)}-\frac{1}{p^{8}(s+1)}\right] \\
& =\frac{1}{5 !} x^{5} e^{-t}+\frac{1}{7 !} x^{7} e^{-t} .
\end{aligned}
$$

We see that the second term in $u_{0}$ and the first terms in $u_{1}$ becomes zero, keeping the non noise terms in $u_{0}$ obtain the exact solution of $\mathrm{Eq}(22)$ as follow

$$
u(x, t)=x e^{-t}
$$

Example 2. Consider the following nonlinear partial differential equation [6]

$$
u_{x x}-u u_{t t}=-x^{2} e^{-2 t}
$$

with initial conditions

$$
u(0, t)=0, \quad u_{x}(0, t)=e^{-t} .
$$


By applying the aforesaid method subject to the initial condition, we have

$$
U(p, s)=\frac{1}{p^{2}(s+1)}-\frac{2}{p^{5}(s+2)}+\frac{1}{p^{2}} L_{x} L_{t}\left[u u_{t t}\right]
$$

On using inverse double Laplace transform

$$
u(x, t)=x e^{-t}-\frac{x^{4} e^{-2 t}}{12}+L_{p}^{-1} L_{s}^{-1}\left[\frac{1}{p^{2}} L_{x} L_{t}\left[u u_{t t}\right]\right]
$$

by using $\operatorname{Eq}(17)$ we get

$$
\sum_{n=0}^{\infty} u_{n}(x, t)=x e^{-t}-\frac{x^{4} e^{-2 t}}{12}+L_{p}^{-1} L_{s}^{-1}\left[\frac{1}{p^{2}} L_{x} L_{t}\left[\sum_{n=0}^{\infty} A_{n}\right]\right]
$$

where $A_{n}$ are Adomian polynomials that represent

$$
u u_{t t}=\sum_{n=0}^{\infty} A_{n}
$$

The first few components of Adomian polynomials, are given by

$$
\begin{aligned}
& A_{0}=\left(u_{0}\right)\left(u_{0}\right)_{t t} \\
& A_{1}=\left(u_{0}\right)\left(u_{1}\right)_{t t}+\left(u_{1}\right)\left(u_{0}\right)_{t t} \\
& A_{2}=\left(u_{0}\right)\left(u_{2}\right)_{t t}+\left(u_{1}\right)\left(u_{1}\right)_{t t}+\left(u_{2}\right)\left(u_{0}\right)_{t t}
\end{aligned}
$$

The recursive relation is given below

$$
\begin{gathered}
u_{0}(x, t)=x e^{-t}-\frac{1}{12} x^{4} e^{-2 t} \\
u_{n+1}(x, t)=L_{p}^{-1} L_{s}^{-1}\left[\frac{1}{p^{2}} L_{x} L_{t}\left[\sum_{n=0}^{\infty} A_{n}\right]\right]
\end{gathered}
$$

The other components of the solution can easily found by using above recursive relation

$$
\begin{aligned}
u_{1}(x, t) & =L_{p}^{-1} L_{s}^{-1}\left[\frac{1}{p^{2}} L_{x} L_{t}\left[\sum_{n=0}^{\infty} A_{0}\right]\right] \\
& =L_{p}^{-1} L_{s}^{-1}\left[\frac{1}{p^{2}} L_{x} L_{t}\left[x^{2} e^{-2 t}-\frac{5}{12} x^{5} e^{-3 t}+\frac{1}{36} x^{8} e^{-4 t}\right]\right] \\
& =\frac{1}{12} x^{4} e^{-2 t}-\frac{5}{12 \times 42} x^{7} e^{-3 t}+\frac{1}{36 \times 90} x^{10} e^{-4 t}
\end{aligned}
$$

It is obvious that the self-canceling "noise" terms appear between various components. Canceling the second term in $u_{0}$ and the first terms in $u_{1}$, keeping the non noise terms in $u_{0}$ yields the exact solution of Eq(32) given by

$$
u(x, t)=x e^{-t}
$$

Example 3. Consider nonlinear partial differential equation [7]

$$
u_{x x}-u_{x} u_{t t}=-x+u
$$


with initial conditions

$$
u(0, t)=\sin t, \quad u_{x}(0, t)=1
$$

Applying the double and single Laplace transform we get

$$
U(p, s)=\frac{1}{p\left(s^{2}+1\right)}+\frac{1}{p^{2} s}-\frac{1}{p^{4} s}+\frac{1}{p^{2}} L_{x} L_{t}\left[u_{x} u_{t t}+u\right]
$$

The inverse of double Laplace transform implies that

$$
u(x, t)=\sin t+x-\frac{x^{3}}{3 !}+L_{p}^{-1} L_{s}^{-1}\left[\frac{1}{p^{2}} L_{x} L_{t}\left[u_{x} u_{t t}+u\right]\right]
$$

We decompose the solution as an infinite sum given below

$$
\sum_{n=0}^{\infty} u_{n}(x, t)=\sin t+x-\frac{x^{3}}{3 !}+L_{p}^{-1} L_{s}^{-1}\left[\frac{1}{p^{2}} L_{x} L_{t}\left[\sum_{n=0}^{\infty} A_{n}+\sum_{n=0}^{\infty} u_{n}(x, t)\right]\right]
$$

The nonlinear term is handled with the help of Adomian polynomials [7] as

$$
u_{x} u_{t t}=\sum_{n=0}^{\infty} A_{n}
$$

The recursive relation is given below

$$
\begin{aligned}
u_{0}(x, t) & =\sin t+x-\frac{x^{3}}{3 !} \\
u_{1}(x, t) & =L_{p}^{-1} L_{s}^{-1}\left[\frac{1}{p^{2}} L_{x} L_{t}\left[\sum_{n=0}^{\infty} A_{0}+\sum_{n=0}^{\infty} u_{0}(x, t)\right]\right] \\
u_{n+1}(x, t) & =L_{p}^{-1} L_{s}^{-1}\left[\frac{1}{p^{2}} L_{x} L_{t}\left[\sum_{n=0}^{\infty} A_{n}+\sum_{n=0}^{\infty} u_{n}(x, t)\right]\right]
\end{aligned}
$$

The other components of the solutions can be easily found by using above recursive relation

$$
\begin{aligned}
u_{1}(x, t) & =L_{p}^{-1} L_{s}^{-1}\left[\frac{1}{p^{2}} L_{x} L_{t}\left[\left(u_{0}\right)_{x}\left(u_{0}\right)_{t t}+u_{0}(x, t)\right]\right] \\
& =L_{p}^{-1} L_{s}^{-1}\left[\frac{1}{p^{2}} L_{x} L_{t}\left[\frac{x^{2}}{2} \sin t+x-\frac{x^{3}}{3 !}\right]\right] \\
& =L_{p}^{-1} L_{s}^{-1}\left[\frac{1}{p^{5}\left(s^{2}+1\right)}+\frac{1}{p^{4} s}-\frac{1}{p^{6} s}\right] \\
& =\frac{x^{4}}{4 !} \sin t+\frac{x^{3}}{3 !}-\frac{x^{5}}{5 !}
\end{aligned}
$$

It is important to recall here that the noise terms appear between the components $u_{0}(x, t)$ and $u_{1}(x, t)$, where the noise terms are those pairs of terms that are identical but carrying opposite signs. More precisely, the noise terms $\pm \frac{x^{3}}{3 !}$ between the components $u_{0}(x, t)$ and $u_{1}(x, t)$ can be cancelled and the remaining terms of $u_{0}(x, t)$ still satisfy the equation. Therefore, the exact solution is given by

$$
u(x, t)=\sum_{n=0}^{\infty} u_{n}(x, t)=\sin t+x
$$


Example 4. Consider one dimensional nonlinear wave-like equation [8]

$$
u_{t t}=x^{2} \frac{\partial}{\partial x}\left(u_{x} u_{x x}\right)-x^{2}\left(u_{x x}\right)^{2}-u
$$

with the initial conditions

$$
u(x, 0)=0, u_{t}(x, 0)=x^{2}
$$

Taking the double Laplace transform there is ( denoted by $L_{2}$ ) on both sides of Eq(44) we obtain

$$
s^{2} U(p, s)-s U(p, 0)-\frac{\partial U(p, 0)}{\partial t}=L_{x} L_{t}\left(x^{2} \frac{\partial}{\partial x}\left(u_{x} u_{x x}\right)-x^{2}\left(u_{x x}\right)^{2}-u\right) .
$$

Applying the initial conditions given in $\mathrm{Eq}(45)$, we have

$$
U(p, s)=\frac{2}{p^{3} s^{2}}+\frac{1}{s^{2}} L_{x} L_{t}\left(x^{2} \frac{\partial}{\partial x}\left(u_{x} u_{x x}\right)-x^{2}\left(u_{x x}\right)^{2}-u\right) .
$$

Operating the inverse double Laplace transform on both sides of Eq(47), we have

$$
u(x, t)=x^{2} t+L_{2}^{-1}\left(\frac{1}{s^{2}} L_{x} L_{t}\left(x^{2} \frac{\partial}{\partial x}(N u)-x^{2}(M u)-u\right)\right)
$$

where

$$
N u=u_{x} u_{x x}=\sum_{n=0}^{\infty} A_{n}, \quad M u=\left(u_{x x}\right)^{2}=\sum_{n=0}^{\infty} B_{n}
$$

Using Eq(49) we obtain Adomian's polynomials as follows

$$
\begin{aligned}
& A_{0}=\left(u_{0}\right)_{x}\left(u_{0}\right)_{x x} \\
& A_{1}=\left(u_{0}\right)_{x}\left(u_{1}\right)_{x x}+\left(u_{1}\right)_{x}\left(u_{0}\right)_{x x} \\
& A_{2}=\left(u_{0}\right)_{x}\left(u_{2}\right)_{x x}+\left(u_{1}\right)_{x}\left(u_{1}\right)_{x x}+\left(u_{2}\right)_{x}\left(u_{0}\right)_{x x}
\end{aligned}
$$

and

$$
B_{0}=\left(u_{0}\right)_{x x}^{2}, B_{1}=2\left(u_{0}\right)_{x x}\left(u_{1}\right)_{x x}, B_{2}=\left(u_{1}\right)_{x x}^{2}+2\left(u_{0}\right)_{x x}\left(u_{2}\right)_{x x}
$$

Starting with $u_{0}(x, t)=x^{2} t$ and using

$$
u_{n_{+1}}(x, t)=L_{p}^{-1} L_{s}^{-1}\left(\frac{1}{s^{2}} L_{x} L_{t}\left(x^{2} \frac{\partial}{\partial x} \sum_{n=0}^{\infty} A_{n}\right)\right)-L_{p}^{-1} L_{s}^{-1}\left(\frac{1}{s^{2}} L_{x} L_{t}\left(x^{2} \sum_{n=0}^{\infty} B_{n}\right)\right)-L_{p}^{-1} L_{s}^{-1}\left(\frac{1}{s^{2}} L_{x} L_{t} \sum_{n=0}^{\infty}\left(u_{n}\right)\right)
$$

We can obtain

$$
\begin{aligned}
u_{1}(x, t) & =L_{p}^{-1} L_{s}^{-1}\left(\frac{1}{s^{2}} L_{x} L_{t}\left(x^{2} \frac{\partial}{\partial x}\left(A_{0}\right)\right)\right)-L_{p}^{-1} L_{s}^{-1}\left(\frac{1}{s^{2}} L_{x} L_{t}\left(x^{2}\left(B_{0}\right)\right)\right)-L_{p}^{-1} L_{s}^{-1}\left(\frac{1}{s^{2}} L_{x} L_{t}\left(u_{0}\right)\right) \\
& =-L_{p}^{-1} L_{s}^{-1}\left(\frac{1}{s^{2}} L_{x} L_{t}\left(x^{2} t\right)\right)=-L_{p}^{-1} L_{s}^{-1}\left(\frac{2}{p^{3} s^{4}}\right)=-\frac{x^{2} t^{3}}{3 !}
\end{aligned}
$$




$$
\begin{gathered}
u_{2}(x, t)=L_{p}^{-1} L_{s}^{-1}\left(\frac{1}{s^{2}} L_{x} L_{t}\left(x^{2} \frac{\partial}{\partial x}\left(A_{1}\right)\right)\right)-L_{p}^{-1} L_{s}^{-1}\left(\frac{1}{s^{2}} L_{x} L_{t}\left(x^{2}\left(B_{1}\right)\right)\right)-L_{p}^{-1} L_{s}^{-1}\left(\frac{1}{s^{2}} L_{x} L_{t}\left(u_{1}\right)\right) \\
=-L_{p}^{-1} L_{s}^{-1}\left(\frac{1}{s^{2}} L_{x} L_{t}\left(-\frac{x^{2} t^{3}}{3 !}\right)\right)=L_{p}^{-1} L_{s}^{-1}\left(\frac{2}{p^{3} s^{6}}\right)=\frac{x^{2} t^{5}}{5 !} \\
u_{3}(x, t)=-\frac{x^{2} t^{7}}{7 !}
\end{gathered}
$$

On using Eq(14), we have

$$
u(x, t)=\sum_{n=0}^{\infty} u_{n}(x, t)=x^{2}\left(t-\frac{t^{3}}{3 !}+\frac{t^{5}}{5 !}-\frac{t^{7}}{7 !}+\ldots\right)
$$

The exact solution is $u(x, t)=x^{2} \sin t$.

\section{Competing interests}

The authors declare that they have no competing interests.

\section{Authors' contributions}

All authors have contributed to all parts of the article. All authors read and approved the final manuscript.

\section{References}

[1] AM Wazwaz, A new technique for calculating adomian polynomials for nonlinear polynomials. Appl. Math. Comput. 111( 2002) $33-51$.

[2] A. Kiliçman and H. E. Gadain, "On the applications of Laplace and Sumudu transforms," Journal of the Franklin Institute, vol. 347, no. 5, pp. 848-862, 2010.

[3] H. Eltayeb and A. Kiliçman, A Note on Double Laplace Transform and Telegraphic Equations. Abstract and Applied Analysis, Volume 2013 (2013), Article ID 932578, 6 pages.

[4] A. Kiliçman and H. Eltayeb, A note on defining singular integral as distribution and partial differential equation with convolution term, Math. Comput. Modelling 49 (2009) 327-336.

[5] H. Eltayeb and A. Kiliçman, A Note on Solutions of Wave, Laplace's and Heat Equations with Convolution Terms by Using Double Laplace Transform: Appl, Math, Lett. 21 (12) (2008), 1324-1329.

[6] D. Kaya and M. Inc. On the Solution of the Nonlinear Wave Equation by the Decomposition Method, Bull. Malaysian Math. Soc. (Second Series) 22 (1999) 151-155.

[7] A.M. Wazwaz, Partial differential equations methods and applications, Netherland Balkema Publisher, 2002.

[8] Mohammad Ghoreishi. Adomian Decomposition Method (ADM) for Nonlinear Wave-like Equations with Variable Coefficient, Applied Mathematical Sciences, Vol. 4, 2010, no. 49, 2431 - 2444. 